\title{
Focus Detection from Digital In-Line Holograms Based on Spectral $l_{1}$ Norms
}

\author{
Weichang Li \\ Applied Ocean Physics and Engineering Department, Woods Hole Oceanographic Institution \\ Woods Hole, MA 02543 \\ Nick C. Loomis \\ Department of Mechanical Engineering, Massachusetts Institute of Technology \\ Cambridge, MA 02139 \\ Qiao Hu and Cabell S. Davis \\ Department of Biology, Woods Hole Oceanographic Institution \\ Woods Hole, MA 02543
}

In this paper a rapid focus detection technique is developed for objects imaged using digital in-line holograms. It differs from previous approaches in that it is based directly on the spectral content of the object images and does not need a full reconstruction of the actual images. It is based on new focus metrics defined as the $l_{1}$ norms of the object spectral components associated with the real and imaginary parts of the reconstruction kernel. Furthermore, these $l_{1}$ norms can be computed efficiently in the frequency domain using a polar coordinate system, yielding a drastic speedup of about two orders of magnitude compared with image-based focus detection. The subsequent reconstruction, when done selectively over these detected focus distances, leads to significant computational savings. Focus detection results from holograms of plankton are demonstrated showing that the technique is both accurate and robust.

OCIS codes: 090.0090, 070.6020, 100.3010, 100.6890, 110.3000, 170.3010 


\section{Introduction}

Digital holographic imaging has been increasingly used in a broad spectrum of applications, such as holographic microscopy [1], plankton sampling [2-4] and holographic particle image velocimetry (HPIV) [5-7]. Computational reconstruction from digital in-line holograms usually generates a sequence of 2D images along the axial direction [8,9]. In general, a large number of such object image planes are reconstructed first, upon which post processing is applied to extract application specific parameters such as the precise object axial and transverse location, individual object morphology and large-scale object distributions. This reconstruction process is typically time-consuming, especially when a relatively large volume is reconstructed at high resolution. In most application scenarios, however, the distribution of objects is relatively sparse and only a few of the reconstructed image planes contain infocus objects. The majority of reconstructed images often do not contain any focused object, yet they consume most of the computation. The reconstruction can be accelerated markedly if the focus distances of the objects could be known a priori within some small range. Thus there is a pressing need for effective focus-detection techniques capable of locating the focus distances efficiently prior to the actual reconstruction.

Various focus analysis techniques have been previously reported [10-13]. Most of them extract the focus distances from the sequence of reconstructed images based on certain criteria. Thus the reconstruction is done blindly without knowing the focus distances, and the focus analysis is carried out as a post-processing step. For instance, Yu et al. [13] used a thresholded edge-detector to pick out the focused layer which then is subtracted from the object spectrum. By permuting the order of focus layers and carrying out multiple reconstruction cycles, they concluded that the average of each focus layer image has an improved quality. Clearly this scheme of iterative edge-detection and subtraction with averaging over multiple reconstructions is computationally expensive and the simple thresholded edge-detection may not always yield optimal results. More recently Liebling and Unser [11] proposed to compute the decomposition of the object image in terms of the wavelet like Fresnelet bases and then find the focus distance by maximizing a sharpness metric related to the sparsity of the decomposition coefficients. The hypothesis is that the Fresnelet coefficients for a focused image are sparse. However, although the Fresnelet sparsity-based technique is robust to noise, the resulting resolution is not satisfactory compared with other approaches such as the Laplacian sharpness metric. The recent work by Dubois et al. [10] is closely related to this paper. It had shown that the integration of the amplitude modulus of the reconstructed object image is minimized when an object with real positive amplitude is in focus and maximized when a pure phase object is in focus. A focus plane detection method was proposed based on this amplitude analysis.

While these previous methods can be quite useful for automatic location of objects within 
the sequence of images, they all require full reconstruction of the object images and are not suited for rapid, computationally-efficient applications.

In this paper, a new focus detection technique is developed for objects with real amplitude captured in digital in-line holograms. It differs from all the previous approaches in that it is based directly on the spectral content of the object images, and hence does not require a full reconstruction of the images. It is based on new focus metrics defined as the $l_{1}$ norms of the reconstructed object spectral components associated with the real and imaginary parts of the reconstruction kernel. Furthermore, these $l_{1}$ norms can be computed efficiently in the frequency-domain using a polar coordinate system. The subsequent reconstruction, when done selectively over these detected focus distances, yields significant computational savings.

The remainder of the paper is organized as follows. Section 2 formulates the recording and reconstruction procedures of a digital in-line hologram. Section 3 defines the new focus metrics and presents the focus detection technique. The focus behavior also is explained in terms of spatial smoothing and frequency modulation. An efficient way of computing the focus metrics using polar coordinates is described in Section 4. Section 5 shows experimental results obtained by applying the new focus detection technique to plankton holograms. Both individual hologram cases as well as statistical results obtained from processing a set of holograms are included. Finally, conclusions follow in Section 6. Throughout the paper, ** denote 2D convolution; $\mathcal{F}$ and $\mathcal{F}^{-1}$ denotes the 2D forward and inverse Fourier transform, respectively; Capital letters represent Fourier Transformed variables, and \|\|$_{1}$ denotes the $l_{1}$ norm.

\section{Hologram Recording and Reconstruction}

Consider an object plane located at distance $z$ from and parallel to the hologram (Figure 1 , represented by a field distribution $a(x, y)$. The object wave field at the hologram plane, denoted by $o(x, y)$, can be written in terms of $a(x, y)$ as [14]

$$
\begin{gathered}
o(x, y)=h(x, y ; z) * * a(x, y) \\
O\left(f_{x}, f_{y}\right)=H\left(f_{x}, f_{y} ; z\right) A\left(f_{x}, f_{y}\right)
\end{gathered}
$$

where $h(x, y ; z)$ is the kernel obtained from the free-space Greens function associated with a point source at $(x, y, z)$ and $H\left(f_{x}, f_{y} ; z\right)=\mathcal{F}(h(x, y ; z))$ is the corresponding transfer function. $O\left(f_{x}, f_{y}\right)$ and $A\left(f_{x}, f_{y}\right)$ denote the 2D Fourier transformation of $o(x, y)$ and $a(x, y)$, respectively. 
The kernel and the transfer function are given by [14]:

$$
\begin{aligned}
h(x, y ; z) & =\frac{1}{j \lambda} \frac{e^{j k\left(x^{2}+y^{2}+z^{2}\right)^{1 / 2}}}{\left(x^{2}+y^{2}+z^{2}\right)^{1 / 2}} \\
H\left(f_{x}, f_{y} ; z\right) & = \begin{cases}e^{-j k z\left[1-\left(\lambda f_{x}\right)^{2}-\left(\lambda f_{y}\right)^{2}\right]^{1 / 2}} & \sqrt{f_{x}^{2}+f_{y}^{2}}<\frac{1}{\lambda} \\
0 & \text { otherwise. }\end{cases}
\end{aligned}
$$

where $k \triangleq \frac{2 \pi}{\lambda} . H\left(f_{x}, f_{y} ; z\right)$ is bandlimited within a disc $\sqrt{f_{x}^{2}+f_{y}^{2}}<1 / \lambda$. Note that the practical bandwidth, determined by several factors such as the finite hologram aperture and the camera pixel size (the sample frequency), is usually much smaller than $1 / \lambda$. However, the above bandwidth expression can be easily generalized to accommodate those limitations by replacing $1 / \lambda$ with an appropriate cutoff frequency. Unless explicitly specified, it is always assumed that $f_{x}$ and $f_{y}$ are within the above bandwidth disc throughout the paper.

The in-line hologram, denoted by $i(x, y)$, is the intensity of the sum of the reference wave and the object wave incident on the hologram plane:

$$
\begin{aligned}
i(x, y) & =|1+o(x, y)|^{2} \\
& =o(x, y)+o^{*}(x, y)+|o(x, y)|^{2}+1 .
\end{aligned}
$$

Here the reference wave is a plane-wave and represented simply by unity after normalization. Hereafter the DC term and the negligible nonlinear term will be dropped. The retained two linear terms are denoted by $\tilde{i}(x, y)$ :

$$
\tilde{i}(x, y)=o(x, y)+o^{*}(x, y) .
$$

Let $\tilde{I}\left(f_{x}, f_{y}\right) \triangleq \mathcal{F}(\tilde{i}(x, y))$. Since $\mathcal{F}\left(o^{*}(x, y)\right)=O^{*}\left(-f_{x},-f_{y}\right)$, it follows that

$$
\tilde{I}\left(f_{x}, f_{y}\right)=O\left(f_{x}, f_{y}\right)+O^{*}\left(-f_{x},-f_{y}\right) .
$$

Generally, during reconstruction, a sequence of object images are computed over a range of distances. The object image reconstructed at one such distance $\widehat{z}$ is given by

$$
\widehat{a}(x, y ; \widehat{z})=h^{*}(x, y ; \widehat{z}) * * \tilde{i}(x, y),
$$

or in frequency domain,

$$
\widehat{A}\left(f_{x}, f_{y} ; \widehat{z}\right)=H^{*}\left(f_{x}, f_{y} ; \widehat{z}\right) \tilde{I}\left(f_{x}, f_{y}\right)
$$

since $\mathcal{F}\left(h^{*}(x, y ; \widehat{z})\right)=H^{*}\left(-f_{x},-f_{y} ; \widehat{z}\right)=H^{*}\left(f_{x}, f_{y} ; \widehat{z}\right)$. 


\section{Focus Detection}

\section{A. Image Sparsity Measure}

It was shown recently [10] that, in the absence of twin-image component, i) the energy and the integral of amplitude of the reconstructed object image are invariant to the reconstruction distance; ii) the integral of the amplitude modulus is minimized at the focused distance for object with real positive amplitude and is maximized at the focused distance for pure phase objects. That is

$$
z_{\text {focus }}= \begin{cases}\arg \min _{\widehat{z}} \int_{x, y}|\widehat{a}(x, y ; \widehat{z})| d x d y & \text { if } a(x, y) \text { is real and positive } \\ \arg \max _{\widehat{z}} \int_{x, y}|\widehat{a}(x, y ; \widehat{z})| d x d y & \text { if } a(x, y) \text { is pure phase. }\end{cases}
$$

Although not explicitly stated [10], the integral of the amplitude modulus is in fact exactly the $l_{1}$ norm of the reconstructed object image, i.e.

$$
\|\widehat{a}(x, y ; \widehat{z})\|_{1}=\int_{x, y}|\widehat{a}(x, y ; \widehat{z})| d x d y .
$$

Therefore the results (10) are equivalent to saying that the $l_{1}$ norm of the reconstructed object image is minimized when a real positive object is in focus and maximized when a pure phase object is in focus. Strictly speaking, the degree of sparseness of a signal is quantified by its $l_{0}$ norm, which is the total number of the nonzero signal components. The $l_{1}$ norm is a convex relaxation of the $l_{0}$ norm and is often used due to its analytical tractability.

Although this spatial sparseness as a focus metric is analytically appealing, it is based on the sequence of reconstructed images which need to be computed over a range of distances. Therefore it is not suitable for the purpose mentioned in the introduction section. The new frequency-domain based focus detection technique, developed in the next section for object with real amplitude, is based on focus metrics defined directly in the frequency domain and can be computed prior to the reconstruction.

\section{B. Spectral $l_{1}$ Norm Focus Metrics}

Hereafter $a(x, y)$ is assumed real, hence $A\left(f_{x}, f_{y}\right)=A^{*}\left(-f_{x},-f_{y}\right)$.

Denote the real and imaginary parts of $H\left(f_{x}, f_{y} ; z\right)$ by $H_{c}\left(f_{x}, f_{y} ; z\right)$ and $H_{s}\left(f_{x}, f_{y} ; z\right)$ respectively. Thus,

$$
\begin{aligned}
& H_{c}\left(f_{x}, f_{y} ; z\right) \triangleq \cos \left(k r_{f} z\right) \\
& H_{s}\left(f_{x}, f_{y} ; z\right) \triangleq-\sin \left(k r_{f} z\right),
\end{aligned}
$$

where $r_{f} \triangleq\left[1-\left(\lambda f_{x}\right)^{2}-\left(\lambda f_{y}\right)^{2}\right]^{1 / 2}$. In addition, let

$$
\begin{aligned}
& \widehat{A}_{c}\left(f_{x}, f_{y} ; \widehat{z}\right) \triangleq H_{c}\left(f_{x}, f_{y} ; \widehat{z}\right) \tilde{I}\left(f_{x}, f_{y}\right) \\
& \widehat{A}_{s}\left(f_{x}, f_{y} ; \widehat{z}\right) \triangleq H_{s}\left(f_{x}, f_{y} ; \widehat{z}\right) \tilde{I}\left(f_{x}, f_{y}\right) .
\end{aligned}
$$




\section{Definition of the Focus Metrics :}

The new focus metrics are defined in terms of the $l_{1}$ norms of $\widehat{A}_{c}\left(f_{x}, f_{y} ; \widehat{z}\right)$ and $\widehat{A}_{s}\left(f_{x}, f_{y} ; \widehat{z}\right)$ respectively as follows:

$$
\begin{aligned}
& F_{c}(\Delta z ; z) \triangleq\left\|\widehat{A}_{c}\left(f_{x}, f_{y} ; \widehat{z}\right)\right\|_{1} \\
& F_{s}(\Delta z ; z) \triangleq-\left\|\widehat{A}_{s}\left(f_{x}, f_{y} ; \widehat{z}\right)\right\|_{1}
\end{aligned}
$$

Here $\Delta z \triangleq \widehat{z}-z$. An object then is determined to be in focus at an axial distance where $F_{c}(\Delta z ; z)$ and $F_{s}(\Delta z ; z)$ are maximized. This criterion corresponds to the global maximum for a single object and to one of the local maxima for multiple objects.

Consequently,

$$
\begin{aligned}
F_{c}(\Delta z ; z) & =\int\left|\widehat{A}_{c}\left(f_{x}, f_{y} ; \widehat{z}\right)\right| d f_{x} d f_{y} \\
& =\int\left|H_{c}\left(f_{x}, f_{y} ; \widehat{z}\right) \tilde{I}\left(f_{x}, f_{y}\right)\right| d f_{x} d f_{y} \\
F_{s}(\Delta z ; z) & =-\int\left|\widehat{A}_{s}\left(f_{x}, f_{y} ; \widehat{z}\right)\right| d f_{x} d f_{y} \\
& =-\int\left|H_{s}\left(f_{x}, f_{y} ; \widehat{z}\right) \tilde{I}\left(f_{x}, f_{y}\right)\right| d f_{x} d f_{y} .
\end{aligned}
$$

The remainder of this section shows why $F_{c}(\Delta z ; z)$ and $F_{s}(\Delta z ; z)$ can be used as metrics for focus detection as defined above.

Substituting (2) into (7) and using $H^{*}\left(-f_{x},-f_{y} ; \widehat{z}\right)=H^{*}\left(f_{x}, f_{y} ; \widehat{z}\right)$ and $A\left(f_{x}, f_{y}\right)=$ $A^{*}\left(-f_{x},-f_{y}\right)$ yields

$$
\tilde{I}\left(f_{x}, f_{y}\right)=2 H_{c}\left(f_{x}, f_{y} ; z\right) A\left(f_{x}, f_{y}\right) .
$$

Hence

$$
\widehat{A}_{c}\left(f_{x}, f_{y} ; \widehat{z}\right)=A\left(f_{x}, f_{y}\right) C\left(f_{x}, f_{y} ; \Delta z, z\right)
$$

where

$$
C\left(f_{x}, f_{y} ; \Delta z, z\right) \triangleq\left[\cos \left(k r_{f} \Delta z\right)+\cos \left(k r_{f}(2 z+\Delta z)\right)\right]
$$

Similarly,

$$
\begin{aligned}
\widehat{A}_{s}\left(f_{x}, f_{y} ; \widehat{z}\right) & \triangleq H_{s}\left(f_{x}, f_{y} ; \widehat{z}\right) \tilde{I}\left(f_{x}, f_{y}\right) \\
& =-A\left(f_{x}, f_{y}\right) S\left(f_{x}, f_{y} ; \Delta z, z\right) .
\end{aligned}
$$

where

$$
S\left(f_{x}, f_{y} ; \Delta z, z\right) \triangleq\left[\sin \left(k r_{f} \Delta z\right)+\sin \left(k r_{f}(2 z+\Delta z)\right)\right]
$$


Both $C\left(f_{x}, f_{y} ; \Delta z, z\right)$ and $S\left(f_{x}, f_{y} ; \Delta z, z\right)$ are equal to zero outside the bandwidth limit.

$\widehat{A}_{c}\left(f_{x}, f_{y} ; \widehat{z}\right)$ and $\widehat{A}_{s}\left(f_{x}, f_{y} ; \widehat{z}\right)$ are the spectral components of the reconstructed object image associated with the real and imaginary parts of the reconstruction kernel respectively.

Taking the 2D inverse Fourier transform of both sides of (23) and (25) yields

$$
\begin{aligned}
& \widehat{a}_{c}(x, y ; \widehat{z})=a(x, y) * * c(x, y ; \Delta z, z) \\
& \widehat{a}_{s}(x, y ; \widehat{z})=-a(x, y) * * s(x, y ; \Delta z, z)
\end{aligned}
$$

where $\widehat{a}_{c}(x, y ; \widehat{z}) \triangleq \mathcal{F}^{-1}\left(\widehat{A}_{c}\left(f_{x}, f_{y} ; \widehat{z}\right)\right), \widehat{a}_{s}(x, y ; \widehat{z}) \triangleq \mathcal{F}^{-1}\left(\widehat{A}_{s}\left(f_{x}, f_{y} ; \widehat{z}\right)\right)$ and

$$
\begin{aligned}
& c(x, y ; \Delta z, z)=\mathcal{F}^{-1}\left(C\left(f_{x}, f_{y} ; \Delta z, z\right)\right)=\frac{2 \pi}{\lambda^{2}}\left[\operatorname{sinc}\left(k r_{1}\right)+\operatorname{sinc}\left(k r_{2}\right)\right] \\
& s(x, y ; \Delta z, z)=\mathcal{F}^{-1}\left(S\left(f_{x}, f_{y} ; \Delta z, z\right)\right)=j \frac{2 \pi}{\lambda^{2}}\left[\operatorname{cinc}\left(k r_{1}\right)+\operatorname{cinc}\left(k r_{2}\right)\right]
\end{aligned}
$$

and $\operatorname{sinc}(x)=\sin (x) / x, \operatorname{cinc}(x)=\cos (x) / x, \quad r_{1}=\sqrt{x^{2}+y^{2}+\Delta z^{2}}$ and $r_{2}=$ $\sqrt{x^{2}+y^{2}+(\Delta z+2 z)^{2}}$. The second equalities in (29) and (30) are obtained by first expressing $C\left(f_{x}, f_{y} ; \Delta z, z\right)$ and $S\left(f_{x}, f_{y} ; \Delta z, z\right)$ in terms of complex exponential forms similar to $H\left(f_{x}, f_{y} ; z\right)$ and then using the $2 \mathrm{D}$ Fourier transform pair (3)-(4).

For real $a(x, y), \widehat{a}_{c}(x, y ; \widehat{z})$ represents the object image reconstructed at $\widehat{z}$ and $\widehat{A}_{c}\left(f_{x}, f_{y} ; \widehat{z}\right)$ is its spectrum. The second terms in (24) and (29) correspond to the twin-image component.

The functions $c(x, y ; \Delta z, z)$ and $C\left(f_{x}, f_{y} ; \Delta z, z\right)$ characterizes the focus behavior of the object image in the spatial domain and frequency domain, respectively.

Several useful observations follow from (29) and (24):

1. For $\Delta z \ll z, c(x, y ; \Delta z, z)$ is dominated by $\operatorname{sinc}\left(k r_{1}\right)$ (see Figure 2); similarly $s(x, y ; \Delta z, z)$ is dominated by $\operatorname{cinc}\left(k r_{1}\right)$. Hence although the twin-image effect remains visible in the area surrounding the object, as far as the focus behavior is concerned, it is negligible and may be dropped:

$$
\begin{aligned}
& \widehat{a}_{c}(x, y ; \Delta z) \approx a(x, y) * * \frac{2 \pi}{\lambda^{2}} \operatorname{sinc}\left(k r_{1}\right), \\
& \widehat{a}_{s}(x, y ; \Delta z) \approx-a(x, y) * * \frac{2 \pi}{\lambda^{2}} \operatorname{cinc}\left(k r_{1}\right), \\
& \widehat{A}_{c}\left(f_{x}, f_{y} ; \widehat{z}\right) \approx A\left(f_{x}, f_{y}\right) \cos \left(k r_{f} \Delta z\right), \\
& \widehat{A}_{s}\left(f_{x}, f_{y} ; \widehat{z}\right) \approx-A\left(f_{x}, f_{y}\right) \sin \left(k r_{f} \Delta z\right) .
\end{aligned}
$$

2. At $\Delta z=0, \operatorname{sinc}\left(k r_{1}\right)=\operatorname{sinc}\left(k \sqrt{x^{2}+y^{2}}\right)$ is close to but not exactly equal to the Kronecker delta function. This is the result of the band limitation of the propagation transfer function. However, the full width half maximum (FWHM) of $\operatorname{sinc}\left(k \sqrt{x^{2}+y^{2}}\right)$ is proportional to the wavelength and for most applications is well approximated by the Kronecker delta function. 
3. Equation (31) states that the reconstructed object is the output of convolving the true object with $\operatorname{sinc}\left(k r_{1}\right)$ in the spatial domain. As a result, the $l_{1}$ norm of $\widehat{a}_{c}(x, y ; \Delta z)$ is minimal at the focused distance. When the object is out of focus, the manifest of this spatial convolution is well-known to be a set of fringes surrounding the true object and effectively a spatial smoothing of the object image. The convolution distributes the signal energy more broadly. The more out-of-focus an image is, the smoother it becomes, which means a larger $l_{1}$ norm.

To show that both $F_{c}(\Delta z ; z)$ and $F_{s}(\Delta z ; z)$ are maximized only at $\Delta z=0$, it is assumed here that the twin-image effect is insignificant and the approximations (33) and (34) are used. Therefore,

$$
\begin{aligned}
F_{c}(\Delta z ; z) & =\int\left|\widehat{A}_{c}\left(f_{x}, f_{y} ; \widehat{z}\right)\right| d f_{x} d f_{y} \\
& \approx \int\left|A\left(f_{x}, f_{y}\right)\right|\left|\cos \left(k r_{f} \Delta z\right)\right| d f_{x} d f_{y} \\
& \leq \int\left|A\left(f_{x}, f_{y}\right)\right| d f_{x} d f_{y}
\end{aligned}
$$

where the equality holds only when $\Delta z=0$. Similarly,

$$
\begin{aligned}
F_{s}(\Delta z ; z) & =-\int\left|\widehat{A}_{s}\left(f_{x}, f_{y} ; \widehat{z}\right)\right| d f_{x} d f_{y} \\
& \approx-\int\left|A\left(f_{x}, f_{y}\right)\right|\left|\sin \left(k r_{f} \Delta z\right)\right| d f_{x} d f_{y} \\
& \leq 0
\end{aligned}
$$

where the equality holds only when $\Delta z=0$.

Intuitively, equation (33) indicates that $\widehat{A}_{c}\left(f_{x}, f_{y} ; \widehat{z}\right)$ is $A\left(f_{x}, f_{y}\right)$ modulated by $\cos \left(k r_{f} \Delta z\right)$ which, for $\Delta z \neq 0$, is a set of concentric rings in the frequency domain. The radial cross section of $\cos \left(k r_{f} \Delta z\right)$ is a chirp signal with a chirp rate increasing with $\Delta z$. This spectral modulation controls the focus behavior of the reconstructed image in the frequencydomain. As a result of this modulation, the spectrum of the object image reconstructed out of focus becomes sparse as some of the spectral components are set to zero. Within a fixed bandwidth disc, the number of zeros increases with an increased $\Delta z$ as the chirp rate increases. At $\Delta z=0, \cos \left(k r_{f} \Delta z\right)=1$ within the bandwidth disc. Therefore, the $l_{1}$ norm of $\widehat{A}_{c}\left(f_{x}, f_{y} ; \widehat{z}\right)$ is maximized at the focus distance and decreases as the object is reconstructed further out of focus.

Consider an example of a point source object, i.e. $A\left(f_{x}, f_{y}\right)=1$. Then

$$
\begin{aligned}
& \left.F_{c}(\Delta z ; z)\right|_{\text {point source }}=\int\left|C\left(f_{x}, f_{y} ; \Delta z, z\right)\right| d f_{x} d f_{y} \triangleq F_{p, c}(\Delta z ; z) \\
& \left.F_{s}(\Delta z ; z)\right|_{\text {point source }}=-\int\left|S\left(f_{x}, f_{y} ; \Delta z, z\right)\right| d f_{x} d f_{y} \triangleq F_{p, s}(\Delta z ; z)
\end{aligned}
$$


Dropping the twin-image component in both cases,

$$
\begin{aligned}
& F_{p, c} \approx \int\left|\cos \left(k r_{f} \Delta z\right)\right| d f_{x} d f_{y} \\
& F_{p, s} \approx-\int\left|\sin \left(k r_{f} \Delta z\right)\right| d f_{x} d f_{y}
\end{aligned}
$$

It is easy to verify that both are maximized at the focus distance $\widehat{z}=z$.

In the presence of the twin-image, since neither (37) nor (38) has closed form expression, it can be shown numerically that over $\Delta z \in(-z, \infty)$ both $F_{p, c}(\Delta z ; z)$ and $F_{p, s}(\Delta z ; z)$ are maximized at $\Delta z=0$. Figure 3 has plotted the numerical evaluations of $F_{p, c}$ and $F_{p, s}$ for a point object hologram, with a $256 \times 256$ pixel image, $633 \mathrm{~nm}$ wavelength and $7.9375 \mu \mathrm{m}$ pixel size. Both $F_{p, c}(\Delta z ; z)$ (the lower panels) and $F_{p, s}(\Delta z ; z)$ (the upper panels) were evaluated over $\widehat{z} \in[0,100+z]$ for $z=10,55,100 \mathrm{~mm}$ and normalized by the hologram size. The left panel covers the whole range of $\widehat{z} \in[0,100+z]$ while the small panel on the right shows a close-up plot around $\Delta z=0$. In these plots both $F_{p, c}$ and $F_{p, s}$ attains peak response at the actual object distance. The response shown is robust to the twin-image effect.

The calculation of both $F_{c}(\Delta z ; z)$ and $F_{s}(\Delta z ; z)$ involves a 2D Fourier transform of the hologram and for each $\Delta z$ computing the $l_{1}$ norm specified by (19). Compared with a full reconstruction, it avoids the $2 \mathrm{D}$ inverse Fourier transformation at each reconstruction distance. In the next section, a more efficient implementation in the polar coordinate system in the frequency domain is developed, which speeds up the processing time dramatically.

\section{Efficient Implementation in the Polar Coordinate System}

This section develops an efficient algorithm that speeds up the focus metrics computation by exploiting the circular symmetry of both $H_{c}\left(f_{x}, f_{y} ; \widehat{z}\right)$ and $H_{s}\left(f_{x}, f_{y} ; \widehat{z}\right)$.

The integrand of (19) can be separated into

$$
\left|\widehat{A}_{c}\left(f_{x}, f_{y} ; \widehat{z}\right)\right|=\left|H_{c}\left(f_{x}, f_{y} ; \widehat{z}\right) \tilde{I}\left(f_{x}, f_{y}\right)\right|=\left|H_{c}\left(f_{x}, f_{y} ; \widehat{z}\right)\right|\left|\tilde{I}\left(f_{x}, f_{y}\right)\right|
$$

Note that $H_{c}\left(f_{x}, f_{y} ; \widehat{z}\right)$ is circularly symmetric, as seen in (12), and can be rewritten as

$$
H_{c}\left(f_{x}, f_{y} ; \widehat{z}\right)=H_{c}\left(f_{r} ; \widehat{z}\right)
$$

with no angular dependence. Here $f_{r} \triangleq \sqrt{f_{x}^{2}+f_{y}^{2}}$ denotes the radial spatial frequency. This fact can be used to remove one integration if (19) is converted from a Cartesian coordinate system into a polar system. The focus metric becomes

$$
\begin{aligned}
F_{c}(\Delta z ; z) & =\int_{f_{r}=0}^{\infty} \int_{f_{\theta}=0}^{2 \pi}\left|H_{c}\left(f_{r} ; \widehat{z}\right) \tilde{I}\left(f_{r}, f_{\theta}\right)\right| f_{r} d f_{r} d f_{\theta} \\
& =\int_{f_{r}=0}^{\infty}\left|H_{c}\left(f_{r} ; \widehat{z}\right)\right|\left[\int_{f_{\theta}=0}^{2 \pi}\left|\tilde{I}\left(f_{r}, f_{\theta}\right)\right| d f_{\theta}\right] f_{r} d f_{r} .
\end{aligned}
$$


Here, $f_{\theta}$ is the angular spatial frequency.

The inner integration over $\left|\tilde{I}\left(f_{r}, \theta\right)\right|$ is now independent of $H_{c}\left(f_{r} ; \widehat{z}\right)$ and hence $\widehat{z}$. It is a constant for a given hologram that can be precalculated. Thus, the focus metric integration is reduced to a single integration over $f_{r}$ using a polar form. Derivation of $F_{s}$ is similar, replacing $H_{c}$ with $H_{s}$. Table 1 summerizes the proposed algorithm.

1. Calculate $\tilde{I}\left(f_{x}, f_{y}\right), 2 \mathrm{D}$ FFT of the hologram;

2. Calculate $\tilde{I}\left(f_{r}, f_{\theta}\right)$ from $\tilde{I}\left(f_{x}, f_{y}\right)$ via polar coordinate transformation;

3. Calculate $\int_{f_{\theta}=0}^{2 \pi}\left|\tilde{I}\left(f_{r}, f_{\theta}\right)\right| d f_{\theta}$;

4. Calculate $F_{c}(\Delta z ; z)$ according to (43) for $\{\widehat{z}\}=\widehat{z}_{1}, \cdots \widehat{z}_{S}$ and similarly for $F_{s}$;

5. Focus detection via search peaks in $F_{c}$ or $F_{s}$, or a combination of both, over $\{\widehat{z}\}$, obtain a set of detected distances $\left\{\widehat{z}_{d}\right\}$;

6. Selective reconstruction over $\left\{\widehat{z}_{d}\right\}$

Table 1. The Algorithm for spectral $l_{1}$ norm focus metrics calculation in polar coordinate system and selective reconstruction.

The single most pressing challenge for using a polar form involves computing $\tilde{I}\left(f_{r}, \theta\right)$ from $\tilde{I}\left(f_{x}, f_{y}\right)$. The approach taken here is to use a bilinear interpolation to resample $\tilde{I}\left(f_{x}, f_{y}\right)$, which was found to be sufficient performance-wisely while limiting the amount of computation required. Other methods for calculating the polar Fourier transform with increased accuracy have been proposed [15] at the risk of additional computation time and complexity. The $f_{r}$ grid points are oversampled to account for non-regular radial spacing over the rectangular $f_{x}, f_{y}$ grid. The sampling rate in $f_{\theta}$ can be reduced to increase calculation speed. The inner $90 \%$ of the Fourier transform, measured across the diagonal, was used to estimate the polar spatial frequencies in order to capture a portion of the high-frequency information contained in the corners without significantly increasing the error.

\section{Computational Savings:}

The most significant benefit of using $l_{1}$ norms in the frequency domain is fast computation. The initial 2D Fourier transform of an $N \times N$ hologram is a common task and only needs to be done once. The spatial $l_{1}$ norm method point-wise multiplies the spectral coefficients by $H\left(f_{x}, f_{y} ; \widehat{z}\right)$, takes the inverse 2D Fourier transform, and applies a focus measure in the spatial domain for each particular $\widehat{z}$. Its focus measure is the sum of magnitudes of all $N^{2}$ 
elements, assumed to run in approximately $k N^{2}$ time, for some positive number $k$. If $S$ depths are scanned to find the best focus, the total operation count is

$$
o p_{\text {spatial }}=2 N^{2} \log _{2} N+\left(2 \log _{2} N+k+1\right) N^{2} S
$$

Taking the complex magnitudes and summing in the frequency domain avoids the additional inverse Fourier transform, so that the operation count for the $l_{1}$ norm technique in Cartesian frequency domain coordinate system becomes

$$
o p_{\text {freq }}=2 N^{2} \log _{2} N+(k+1) N^{2} S
$$

Discounting the initial Fourier transform (which is not significant compared to other terms when the value of $S$ is large), the method-dependent speedup is then given by

$$
\frac{o p_{\text {spatial }}}{o p_{\text {freq }}}=\frac{2}{k+1} \log _{2} N+1
$$

Typical holograms, sized $512 \times 512$ pixels through $2048 \times 2048$ pixels, have speedups of 2.6 to 3.0 respectively, for $k=10$.

Using the polar transform adds a layer of complexity but significantly boosts computation rates for even small $S$. After the initial Fourier transform, the spectral data is resampled using a bilinear interpolation and the complex magnitudes are summed across one dimension. A depth kernel and additional complex magnitude summation along the remaining dimension are then applied for each sampled depth. Denoting the total number of radial and angular sampling points as $R$ and $\theta$, respectively, and noting that a simple bilinear interpolation takes $6 R \theta$ multiplications, the total operation count using the polar transform method is

$$
o p_{p o l}=2 N^{2} \log _{2} N+(6+k) R \theta+(k+1) R S
$$

Approximating $R \theta \approx N^{2}$ and $R \approx 2 N$, and again discounting the initial Fourier transform common to each method, the speedup is then

$$
\frac{o p_{\text {spatial }}}{o p_{\text {pol }}}=\frac{\left(2 \log _{2} N+k+1\right) N^{2} S}{(k+6) N^{2}+2(k+1) N S}
$$

For $N=1024, S=100$ and $k=10$, the speedup is approximately 167, two orders of magnitude faster than the spatial $l_{1}$ norm based method, and about 60 times faster than the full two-dimensional frequency based $l_{1}$ norm based method.

\section{Experimental Results}

\section{A. Case examples}

The new frequency domain focus metrics defined in (18), (20) were calculated from several real holograms of marine plankton, using both the Cartesian coordinate system implementation and the polar coordinate system via the fast algorithm. A least squares fitting was 
applied to all focus metrics to extract and remove the linear trend. Then the focus metrics were smoothed over plus and minus $1 \mathrm{~mm}$ and normalized to $[0,1]$. In each case, only the product of the resulting $F_{c}$ and $F_{s}$ is plotted in all the figures, denoted as $F_{c a r t}$ and $F_{\text {pol }}$ respectively.

For each hologram, the spatial domain focus metric developed by Dubois et al. [10] was calculated from a sequence of uniformly spaced reconstructions, without removing the twinimage. The results were then sign reversed, then with linear trend removed, smoothed and normalized in a similar fashion as done to the frequency domain metrics, before being plotted as $F_{\text {spa }}$.

Three examples are shown in Figures 4, 5 and 6, corresponding to a single object, two objects and three objects, respectively. In each figure, the portion of the hologram containing the object of interest is shown in the lower left corner. Clockwise starting from the upper left are a sequence of object image planes reconstructed at various distances around the focus points. The plot in the lower middle panel shows the three focus metric curves as a function of the reconstruction distance.

In Figure 4, the object was located at $24 \mathrm{~mm}$ away from the hologram. The hologram was recorded with a collimated He-Ne laser beam of wavelength $633 \mathrm{~nm}$. The digitized hologram's pixel size is $7.9375 \mu \mathrm{m}$. A square cut of size $256 \times 256$ was used for focus detection and reconstruction. The reconstructed images at $z=16,20,24,28,32 \mathrm{~mm}$ are shown and the object is in its best focus at $z=24 \mathrm{~mm}$. Good agreement is observed among $F_{\text {cart }}, F_{\text {pol }}$ and $F_{\text {spa }}$ at this distance.

In Figure 5, the hologram was recorded using a spherical reference beam from a fibercoupled laser source of wavelength $658 \mathrm{~nm}$ and a camera with $9 \mu \mathrm{m}$ pixel size. A $1024 \times 1024$ square cut of the hologram was used for reconstruction as shown at the lower left corner, so that it contained two objects that are laterally close to each other. Images obtained at $z=49,61,73,151,163,175 \mathrm{~mm}$ are shown clockwise in the Figure. The upper copepod is in focus at $z=61 \mathrm{~mm}$ and the lower one at $z=163 \mathrm{~mm}$. The peaks predicted by $F_{\text {cart }}, F_{\text {pol }}$ and $F_{\text {spa }}$ are consistent and match the focus distances found in the reconstructed images.

In Figure 6, three objects, located approximately at $z=45,64,72.5 \mathrm{~mm}$ respectively, were recorded in a single hologram with the same laser source and camera as in Figure 5. A $1024 \times 1024$ square cut of the hologram was used for reconstruction as shown at the lower left corner; clockwise starting from the upper left in the figure are images reconstructed at $z=42,45,48,62,64,67,70,72.5,75 \mathrm{~mm}$, respectively. The copepod on the upper left corner of the hologram is focused at $z=45 \mathrm{~mm}$; the one in the middle is focused at $z=64$ mm. It was oriented such that its body extends in the axial direction. As a result, all focus metrics have broadened responses at $z=64 \mathrm{~mm}$. The copepod at the lower left is in focus at $z=72.5 \mathrm{~mm}$. All three curves are consistent and accurately predict the distances at 
which the objects are in focus. The peaks around $z=85 \mathrm{~mm}$ are associated with some small particles not shown in the figure that are focused at that distance.

\section{B. Statistical results}

In order to statistically verify the accuracy and robustness of the proposed technique, the fast algorithm implemented in the polar coordinate system was integrated into an automatic focus detection and selective reconstruction program which was applied to a set of holograms of plankton. These holograms were taken on August 03, 2005 at MIT's hologram laboratory, and each contained from 10 to 26 large objects. For each hologram, a number of regions of interest, each of size $1024 \times 1024$ pixels, were selected around the objects. The algorithm then calculated the focus metrics for each region over an axial distance range from $30 \mathrm{~mm}$ to $116 \mathrm{~mm}$ at an interval of $1 \mathrm{~mm}$. A simple peak detection algorithm was used to select peaks from the focus metric curves. Full reconstructions were then carried out around these detected axial distances, based on which both the probability of detection $P_{d}$ and probability of false alarms $F_{a}$ were calculated, as shown in Table 2 . The mean of $P_{d}$ is $75.35 \%$ and its standard deviation is $10.49 \%$. The mean of $F_{a}$ is $1.15 \%$ and its standard deviation is $0.24 \%$.

\section{Conclusion}

A new focus detection technique is developed in this paper that can locate the object focus distances prior to a full reconstruction. The focus metrics proposed here are based directly on the spectral flatness of the reconstructed images. The technique has potential in both serving as a stand-alone automatic focus detector and as a preprocessor providing the focus depth distances so that the subsequent reconstruction can be done selectively at these distances, saving significant amount of fruitless computations that are inevitable in a blind reconstruction. The technique is especially attractive due to a highly efficient implementation in the polar coordinate system, promising real-time focus detection. The experimental results based on individual as well as a group of plankton holograms show that the technique is accurate and robust.

\section{Acknowledgements}

W. Li would like to thank J. H. Milgram for his previous support on the topic of digital holographic imaging. N. Loomis thanks J. A. Dominguez-Caballero for his significant work in developing the digital holography system used for capturing the holograms analyzed in this paper. 


\begin{tabular}{|c|c|c|c|c|c|}
\hline hologram no. & totl. object counts & positive detections & false alarms & $P_{d}(\%)$ & $F_{a}(\%)$ \\
\hline 1 & 10 & 9 & 12 & 90 & 1.4 \\
\hline 2 & 12 & 9 & 11 & 75 & 1.1 \\
\hline 3 & 14 & 12 & 16 & 86 & 1.4 \\
\hline 4 & 10 & 8 & 9 & 80 & 1.1 \\
\hline 5 & 14 & 9 & 12 & 64 & 1.0 \\
\hline 6 & 13 & 9 & 15 & 69 & 1.3 \\
\hline 7 & 15 & 14 & 17 & 93 & 1.3 \\
\hline 8 & 16 & 13 & 15 & 81 & 1.1 \\
\hline 9 & 12 & 9 & 10 & 75 & 1.0 \\
\hline 10 & 13 & 10 & 11 & 77 & 1.0 \\
\hline 11 & 18 & 16 & 17 & 89 & 1.1 \\
\hline 12 & 16 & 14 & 22 & 88 & 1.6 \\
\hline 13 & 15 & 11 & 17 & 73 & 1.3 \\
\hline 14 & 15 & 8 & 14 & 53 & 1.1 \\
\hline 15 & 21 & 15 & 19 & 71 & 1.1 \\
\hline 16 & 21 & 14 & 20 & 67 & 1.1 \\
\hline 17 & 22 & 15 & 19 & 68 & 1.0 \\
\hline 18 & 20 & 14 & 26 & 70 & 1.5 \\
\hline 19 & 26 & 20 & 19 & 77 & 0.9 \\
\hline 20 & 25 & 15 & 14 & 60 & 0.7 \\
\hline
\end{tabular}

Table 2. Statistics from experimental results.

$P_{d}=$ positive detections/total object counts;

$F_{a}=$ false alarms $/$ (total object counts $\times$ number of planes per object $)$.

\section{References}

1. D. Carl, B. Kemper, G. Wernicke, and G. Von Bally. Parameter-optimized digital holographic microscope for highresolution living-cell analysis. Appl. Optics., 43:6536-6544, 2004.

2. P. R. Hobson and J. Watson. The principles and practice of holographic recording of plankton. J. Opt. A: Pure Appl. Opt., 4:34-49, 2002.

3. S. K. Jericho, J. Garcia-Sucerquia, W. Xu, M. H. Jericho, and H. J. Kreuzer. Submersible digital in-line holographic microscope. Rev. Sci. Instrum., 77:043706, 2006.

4. W. B. Xu, M. H. Jericho, I. A. Meinertzhagen, and H. J. Kreuzer. Digital in-line holography for biological applications. Proc. Natl. Acad. Sci. U.S.A, 98:11301-11305, 2001.

5. E. Malkiel, J. Sheng, J. Katz, and J. Strickler. The three dimensional flow field generated by a feeding calanoid copepod measured using digital holography. J. Exp. Biol., 206:3657-3666, 2003. 
6. J. Sheng, E. Malkiel, and J. Katz. Digital holographic microscope for measuring threedimensional particle distributions and motions. Appl. Optics, 41:3893-3901, 2006.

7. G. Pan and H. Meng. Digital holography of particle fields: reconstruction by use of complex amplitude. Appl. Optics, 42:827-833, 2003.

8. J. H. Milgram and W. Li. Computational reconstruction of images from holograms. Appl. Optics, 45:853-864, 2002.

9. U. Schnars and W. P. O. Juptner. Digital recording and numerical reconstruction of holograms. Meas. Sci. Technol., 13:R85-R101, 2002.

10. F. Dubois, C. Schockaert, N. Callens, and C. Yourassowsky. Focus plane detection criteria in digital holography microscopy by amplitude analysis. Optics Express, 14:5895$5908,2006$.

11. M. Liebling and M. Unser. Autofocus for digital Fresnel holograms by use of a Fresneletsparsity criterion. J. Opt. Soc. Am. A, 21:12, 2004.

12. A. Thelen, J. Bongartz, G. Dominik, S. Frey, and P. Hering. Iterative focus detection in hologram tomography. J. Opt. Soc. Am. A, 22:1176-1180, 2005.

13. L. Yu and L. Cai. Iterative algorithm with a constraint condition for numerical reconstruction of a three-dimensional object from its hologram. J. Opt. Soc. Am. A, 18:1033-1045, 2001.

14. J. W. Goodman. Introduction to Fourier Optics. McGraw-Hill, 1968.

15. A. Averbuch, R. R. Coifmanb, D.L. Donoho, Elad M., and M. Israeli. Fast and accurate polar Fourier transform. Appl. Comput. Harmon. Anal., 21:146-167, 2006. 


\section{List of Figure Captions}

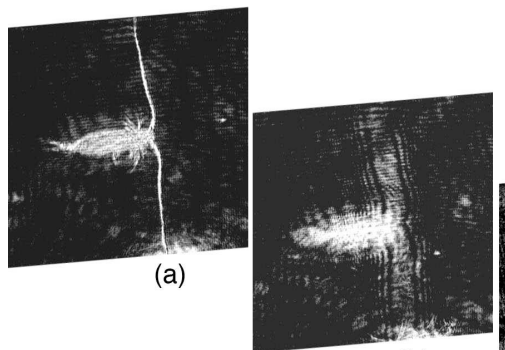

(b)

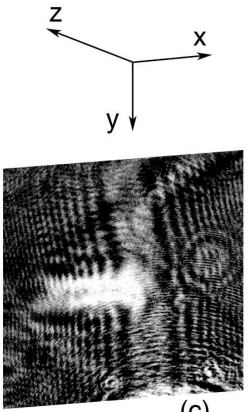

(c)

Fig. 1. Object wavefront propagation and hologram recording: (a) object plane; (b) propagated object field; (c) the hologram.
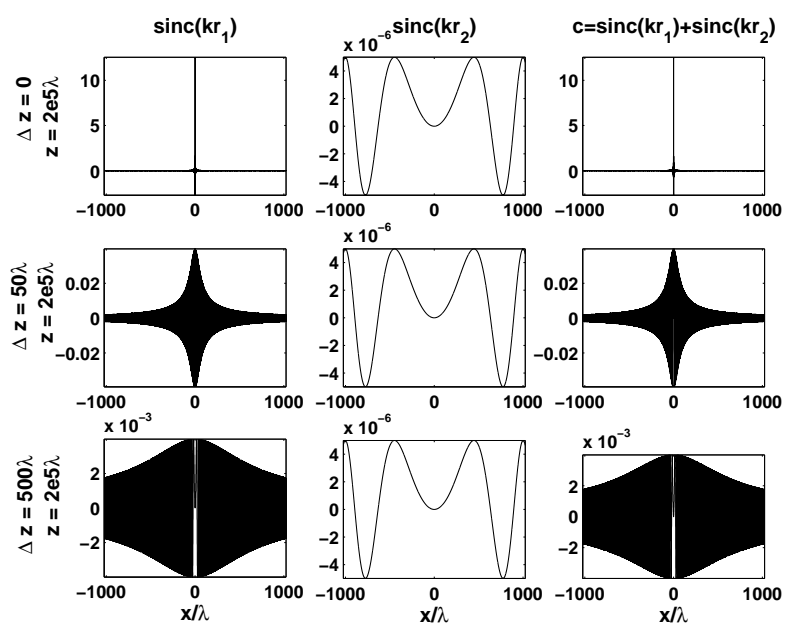

Fig. 2. The spatial convolution kernels $\operatorname{sinc}\left(k r_{1}\right)\left(1\right.$ st column), $\operatorname{sinc}\left(k r_{2}\right)(2 \mathrm{nd}$ column) and $c(x, y ; \Delta z, z)=\operatorname{sinc}\left(k r_{1}\right)+\operatorname{sinc}\left(k r_{2}\right)$ (3rd column), with $z=$

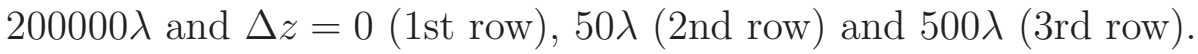



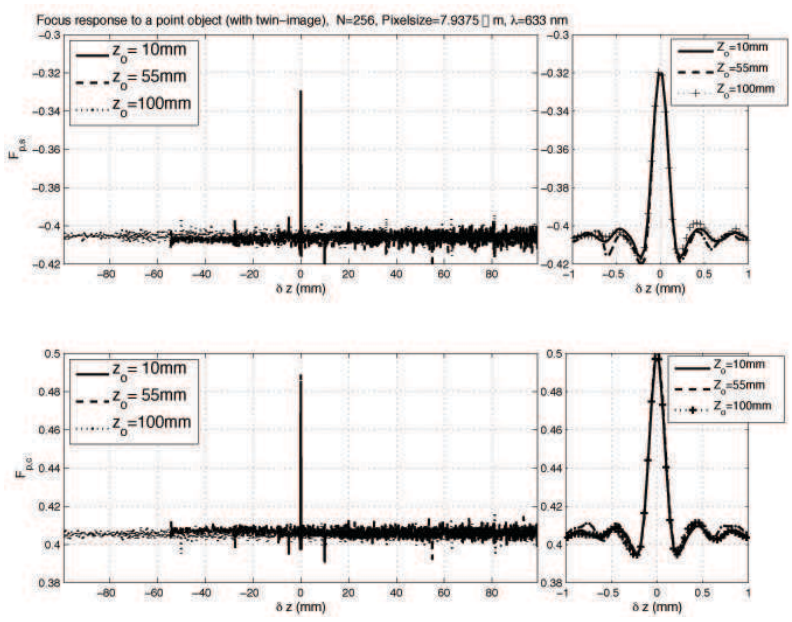

Fig. 3. Given a point object and a hologram of size $256 \times 256$, pixel size $7.9375 \mu \mathrm{m}$ and wavelength $633 \mathrm{~nm}$. (a) $F_{s}(\Delta z ; z)$ (upper panels) and (b) $F_{c}(\Delta z ; z)$ (lower panels) for $\Delta z \in[-z, 100]$ and $z=10,55,100 \mathrm{~mm}$.
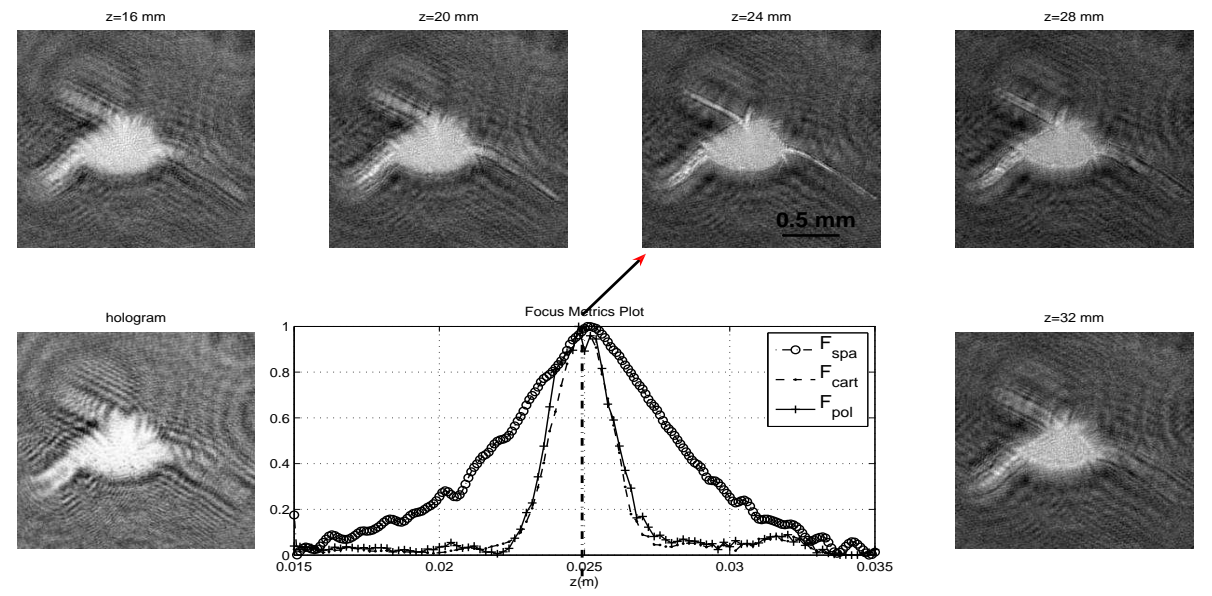

Fig. 4. Focus detection of a single object located at $z=25 \mathrm{~mm}$ from the hologram. The lower left panel shows a square cut of the hologram containing the object; clockwise starting from the upper left are images reconstructed at $z=16,20,24,28,32 \mathrm{~mm}$, respectively. The plot shows the spatial focus metric $F_{s p a}$ (dot-line with circle), the focus metric product in Cartesian system $F_{\text {cart }}$ (dash line with dot) and the focus metric product in polar system $F_{\text {pol }}$ (solid line with cross), as functions of $z$. 

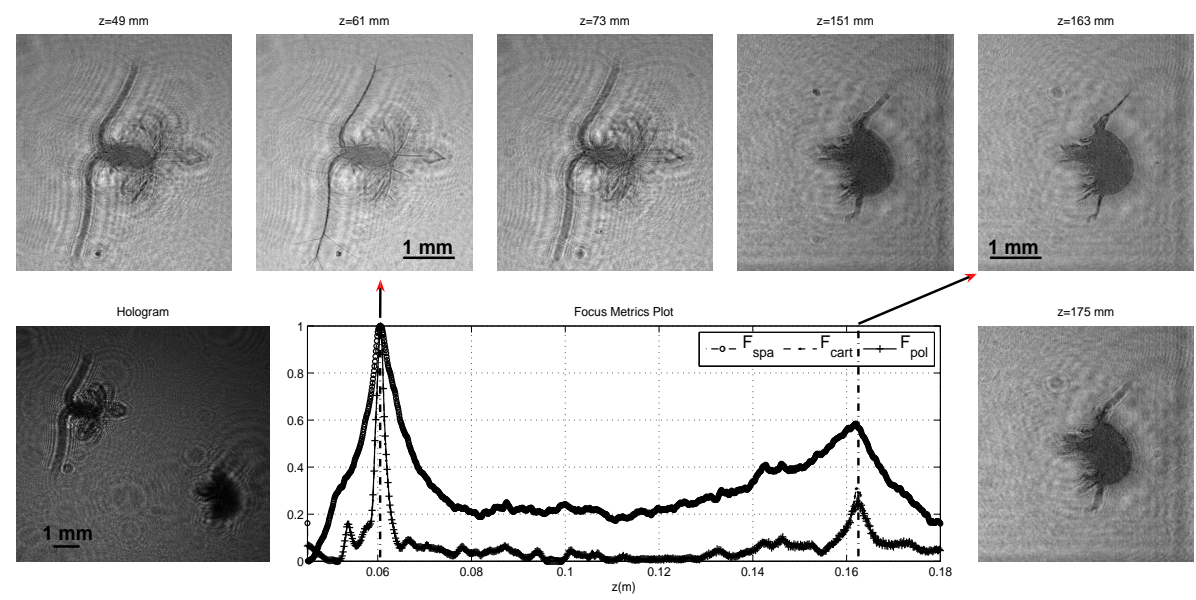

Fig. 5. Focus detection of two objects separated in axial distance, one located at $z=61 \mathrm{~mm}$ and the other $z=163 \mathrm{~mm}$. The lower left panel shows a square cut of the hologram containing both objects; clockwise starting from the upper left are images reconstructed at $z=49,61,73,151,163,175 \mathrm{~mm}$, respectively. The plot shows $F_{s p a}$ (dot-line with circle), the focus metric product in Cartesian system $F_{\text {cart }}$ (dash line with dot) and the focus metric product in polar system $F_{\text {pol }}$ (solid line with cross), as functions of $z$. 


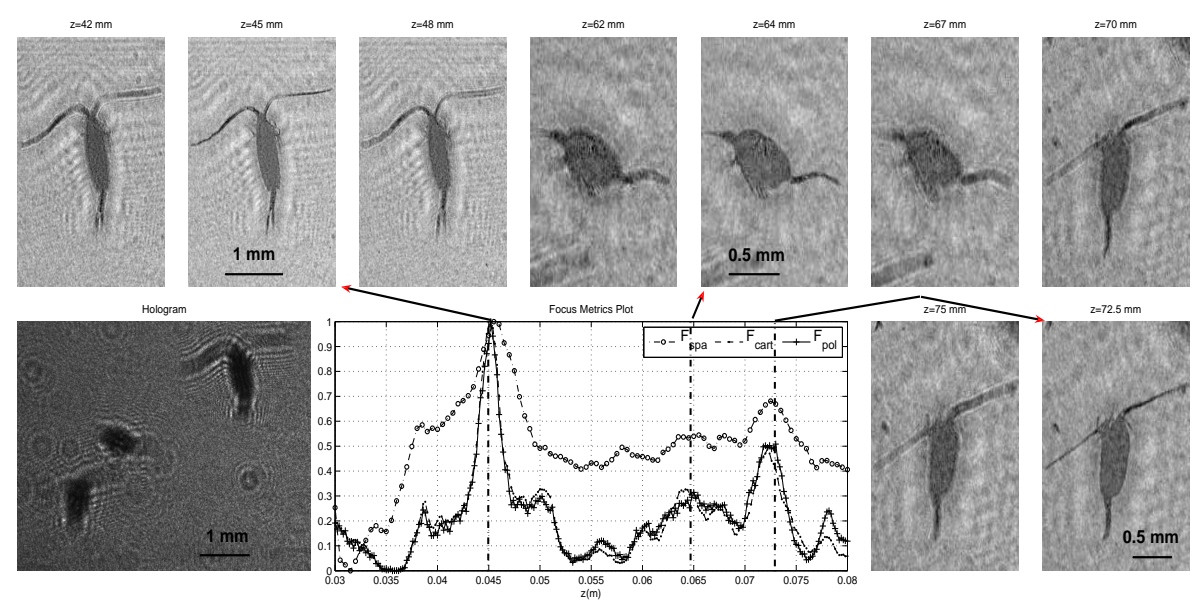

Fig. 6. Focus detection of three objects separated in axial distance, located approximately around $z=45,64,72.5 \mathrm{~mm}$ respectively. The lower left panel shows a square cut of the hologram containing all the objects; clockwise starting from the upper left are images reconstructed at $z=$ 42, 45, 48, 62, 64, 67, 70, 72.5, $75 \mathrm{~mm}$, respectively. The plot shows the spatial focus metric $F_{\text {spa }}$ (dot-line with circle), the focus metric product in Cartesian system $F_{\text {cart }}$ (dash line with dot) and the focus metric product in polar system $F_{\text {pol }}$ (solid line with cross), as functions of $z$. 